\title{
Methylphenidate use among students living in junior on-campus residences of the University of the Free State
}

\author{
${ }^{a}$ Department of Pharmacology, University of the Free State, Bloemfontein, South Africa \\ ${ }^{b}$ Department of Biostatistics, University of the Free State, Bloemfontein, South Africa \\ 'School of Medicine, University of the Free State, Bloemfontein, South Africa \\ *Corresponding author, email: vzylpm@ufs.ac.za
}

PM Van Zyla*, G Joubert ${ }^{b}$, L Fechterc (D), J Grieselc (D), M Nelc, A Honiballc, L Serfontein` and M Diedericksc

Background: The use of methylphenidate as cognitive enhancer is a growing trend among students at tertiary institutions globally. This study aimed to determine the prevalence of methylphenidate use and co-use with alcohol among on-campus residence students of the University of the Free State (UFS).

Methods: For this cross-sectional study, 10 junior residences were randomly selected and 1761 anonymous questionnaires handed out for all students living in these residences during 2015. Data were collected on demographics, use of methylphenidate and co-use of methylphenidate with alcohol.

Results: In total, 585 questionnaires (response rate 33.2\%) were received and analysed. Sixty-six (11.3\%) participants reported past-year use of methylphenidate. While only 18 (27.3\%) of past-year users were diagnosed with ADHD, 44 (66.7\%) obtained their supply through doctors' prescriptions, 21 (31.8\%) from friends without payment, and 4 (6.1\%) bought it from illegal sources. Of the past-year users, $24.2 \%$ had used methylphenidate before consuming alcohol.

Conclusion: Off-label prescribing, diversion of prescriptions and illegal trade in methylphenidate occur among students at the UFS. The frequent co-use of methylphenidate and alcohol may indicate a lack of information on the effects of the medication, rather than deliberate misuse.

Keywords: alcohol co-use, cognitive enhancement, methylphenidate, off-label prescribing, students

\begin{abstract}
Introduction
Attention deficit hyperactivity disorder (ADHD) is the most commonly diagnosed developmental disorder. The Centers for Disease Control and Prevention (CDC) reports a progression in prevalence among the population of the United States, from $10.2 \%$ in 2001 to $14 \%$ in 2013 in boys and from $3.7 \%$ to $5.9 \%$ in girls over the same period. ${ }^{1}$ About $50 \%$ of cases persist into adulthood. ${ }^{2}$ In a large cross-national study, Fayyad et al. ${ }^{3}$ found an adult prevalence of $3.4 \%$, although the authors conceded that this is a conservative figure. There are no published data on the prevalence of ADHD in the South African general population, though it was estimated at around $10 \%$ by $2010 .{ }^{4}$
\end{abstract}

Methylphenidate is still recommended as the first-line choice for treatment of ADHD in children as well as adults, with remarkable effects on ADHD symptoms in classroom settings over the short term, ${ }^{5}$ but long-term effects are not well known. ${ }^{6}$ In addition to being effective for adult ADHD, methylphenidate has been shown to reduce the development of addictive behaviours in the ADHD population, which is known to have a higher propensity for addictive behaviours. ${ }^{7}$ The average dose of methylphenidate for an adult is $20-30 \mathrm{mg} /$ day and the maximum recommended dose is $60 \mathrm{mg} /$ day, yet sometimes higher doses are required for therapeutic effect. ${ }^{8}$

Indiscriminate use of methylphenidate in the absence of ADHD bears the risks of addiction, psychiatric complications and cardiac toxicity when abused. ${ }^{8}$ Toxic effects of methylphenidate appear at excessive doses, with a wide inter-individual variation in response. The combination of dose, route of administration and specific formulation, and co-use with other stimulants or alcohol modifies the toxicity of the drug. Nasal or intravenous administration or combination with other stimulant drugs causes effects similar to amphetamine and cocaine, causing euphoria, delirium, paranoia, hallucinations and aggressive behaviour. ${ }^{8}$ It is therefore classified as a schedule 6 drug in South Africa. ${ }^{9}$

In the wake of the growing awareness of ADHD, the increase in the sales of methylphenidate remains one of the most spectacular in the pharmaceutical industry. Initially introduced to the market in 1952, the production of methylphenidate increased sixfold between 1990 and 2005 . Some $80 \%$ of the worldwide supply was consumed in the United States of America (USA) at the time. ${ }^{10}$ By 2007, 6 million schoolchildren in the USA were using Ritalin ${ }^{\circledast}$. Though the number of Ritalin ${ }^{\oplus}$ users has stabilised, the market for methylphenidate has expanded with the introduction of new brands and formulations. ${ }^{10}$

Similar to the trend in the USA, methylphenidate is increasingly prescribed for school-going children in South Africa. An analysis of the prescription records of a large medical scheme administrator, spanning the prescription records of 24011 medical scheme members, showed that 115 patients received prescriptions for methylphenidate in 2002. ${ }^{11}$ Of these, only 7 (1.5\%) were in the age group 20-29 years, while the peak incidence of methylphenidate prescriptions (50.4\%) occurred in the age group 10-19 years. This figure is not a true reflection of the use of methylphenidate in the South African private health sector, as data on private paying patients were not captured.

Evidence for an increase in non-medical use of methylphenidate, mostly to improve cognitive function, is, however, also escalating among healthy students worldwide. ${ }^{12}$ This generates concerns 
about the safety of methylphenidate, particularly the long-term effects in healthy individuals, and the effects of co-use with alcohol in a population known for binge-drinking behaviour. ${ }^{12}$

\section{Aim of the study}

The objective of this study was to determine the prevalence of methylphenidate use and co-use of methylphenidate with alcohol in students living in junior on-campus residences of the University of the Free State (UFS).

\section{Method}

\section{Study design}

A cross-sectional study design was used.

\section{Target population and sampling}

The target population consisted of students living in junior oncampus residences of the UFS. Junior residences only admit undergraduate students, with $40 \%$ first-year students..$^{13}$ The normal age restriction of 22 years may be exceeded in extraordinary cases where there is no accommodation available in a senior residence. ${ }^{14}$ The 10 randomly selected residences had 1761 students out of a potential 3457 students in 19 residences. The residences included male, female and mixed residences. All residents at the selected facilities were included. Participants had to be registered students of the UFS and living in a junior on-campus residence in 2015.

\section{Questionnaires}

The anonymous, self-administered questionnaire was available in English, and consisted of four sections with a total of 31 questions. Section A consisted of six general demographic questions. Section B (not reported on here) consisted of 11 questions pertaining to alcohol use. Section $C$ consisted of 10 questions pertaining to methylphenidate use, and Section $D$ contained four questions pertaining to the combined use of alcohol and methylphenidate. Most questions were closed ended, but the questionnaire also allowed elaboration under the section 'other' for mentioning different types of cognitiveenhancing drugs. There are no validated instruments for determining non-medicinal use of methylphenidate; the researchers formulated questions based on issues with regard to methylphenidate use highlighted in the literature.

\section{Data collection}

The primaria of each selected residence received an information document regarding the study. Data collection took place during the second half of the year when no examinations were scheduled. The research team visited the residences during house meetings to explain the aim of the study and to seek the residents' cooperation. The 10 residences consisted of 203 single rooms and 779 double rooms. One questionnaire was handed out at each single room and two questionnaires at each double room. A collection box was placed in the foyer of each residence and completed questionnaires were collected daily for a period of two weeks.

\section{Pilot study}

A pilot study was done on five students living in on-campus junior residences that were not selected for the main study. No adjustments to the questionnaire were necessary.

\section{Data analysis}

The completed questionnaires were computerised by the research team on an Excel $^{\circledR}$ spreadsheet (Microsoft Corp, Redmond, WA, (USA). Data analysis was done by the Department of Biostatistics, Faculty of Health Sciences, UFS, using SAS ${ }^{\oplus}$ Version 9.3 (SAS Institute, Cary, NC, USA). Results are summarised by frequencies and percentages (categorical variables) and means, standard deviations (SD) or percentiles (numerical variables, depending on data distribution). The association between the demographic variables age, gender, faculty and year of study and use of methylphenidate was investigated using forward stepwise logistic regression.

\section{Ethical aspects}

Permission to conduct the study (STUD NR 33/2015) was obtained from the Ethics Committee of the Faculty of Health Science, the Vice-Rector Research and the Acting Dean of Student Affairs of the UFS. Participation was voluntary. An information document was attached to each questionnaire. As the questionnaire was anonymous, completion thereof was considered consent.

\section{Results}

Of the 1761 questionnaires handed out, 585 were retrieved and analysed (response rate $33.2 \%$ ). The highest percentage of participants were female (69.6\%) and in their second year of study $(38.1 \%)$ (Table 1$)$. The mean age of the participants was 20.2 years (SD 1.38; range 17-28 years).

Overall, 66 (11.3\%) participants reported using methylphenidate in the past year. Of these 66 students, only 18 (27.3\%) were diagnosed with ADHD. Ritalin ${ }^{\circledast}$ was the most commonly used brand $(n=34,51.5 \%)$, followed by Concerta ${ }^{\circledR}(n=31,47.0 \%)$ Table 1 indicates the past year use of methylphenidate in the demographic subgroups. Male students were twice as likely as

Table 1: Demographic description of participants and past year use of methylphenidate in demographic subgroups

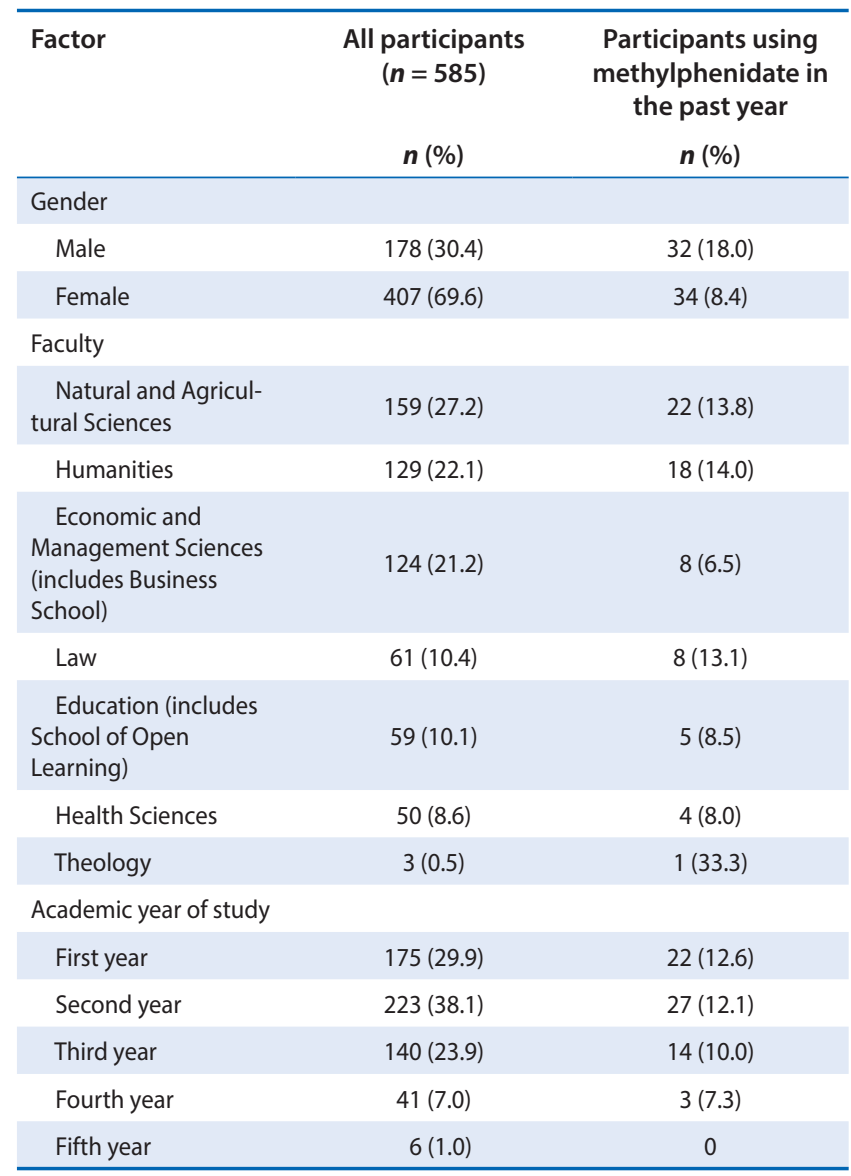


Table 2: Frequency of methylphenidate use among past year users $(n=66)$

\begin{tabular}{lc}
\hline Frequency & $\boldsymbol{n}(\%)^{*}$ \\
\hline Every day & $10(15.2)$ \\
Once a week & $5(7.6)$ \\
Before a test & $30(45.5)$ \\
Once a month & $6(9.1)$ \\
Once a year & $10(15.2)$ \\
Other & $5(7.6)$ \\
\hline
\end{tabular}

*Only one option could be selected.

female students to use methylphenidate. In the logistic regression investigating the listed demographic variables for their independent association with methylphenidate use, gender was the only significant variable with an odds ratio (male versus female) of 2.4 (95\% confidence interval [Cl] $1.4 ; 4.0$ ). Methylphenidate users had a mean age of 20.2 years (SD 1.49) and non-users a mean age of 20.3 years (SD 1.37).

Table 2 reports frequency of methylphenidate use. Nearly half of methylphenidate users ( $n=30 ; 45.5 \%)$ used it before a test, and $15.5 \%(n=10)$ indicated they used it daily.

For methylphenidate use, the median and mode age of onset was 18 years. Most of the participants (75.4\%) were in the age range 16-20 years when they first started using methylphenidate, while $15.4 \%$ were younger than 16 years and $9.3 \%$ were in the age range $21-25$ years. Table 3 reports the situations during which methylphenidate was used, and the effects that users aimed to achieve.

The majority of users used methylphenidate to study better, overwhelmingly to improve concentration. Enhanced confidence and to calm down were highly sought-after effects, while weight loss was not a prominent reason for use. None of the participants used methylphenidate purposely to enhance the effects of other drugs or the acute effects of alcohol.

The majority ( $n=54 ; 81.8 \%)$ of past-year methylphenidate users reported that methylphenidate improved their grades. Only

Table 3: Purpose of methylphenidate use $(n=66)$

\begin{tabular}{|lc|}
\hline Purpose & $n(\%)$ \\
\hline Situations where methylphenidate is used ${ }^{*}$ & $56(84.9)$ \\
\hline Studying before a test & $12(18.2)$ \\
\hline Enhancing general concentration in class & $3(4.6)$ \\
\hline Staying awake in class & $4(6.1)$ \\
\hline Other & \\
\hline Effects that users aim to achieve when using methylphenidate & \\
\hline Improve concentration & $63(95.5)$ \\
\hline Enhanced confidence & $29(43.9)$ \\
\hline Calming down & $29(43.9)$ \\
\hline Focus & $4(6.1)$ \\
\hline Satisfying curiosity & $4(6.1)$ \\
\hline Weight loss & $3(4.6)$ \\
\hline Feeling good & $2(3.0)$ \\
\hline Other & $3(4.6)$ \\
\hline
\end{tabular}

*More than one option could be selected.
Table 4: Source from which methylphenidate was obtained $(n=66)$

\begin{tabular}{lc}
\hline Source & $\boldsymbol{n}^{*}(\%)$ \\
\hline Prescription from general practitioner & $32(48.5)$ \\
Prescription from specialist & $12(18.2)$ \\
Friend (without payment) & $21(31.8)$ \\
Dealer/contact (with payment) & $4(6.1)$ \\
\hline
\end{tabular}

*More than one option could be selected.

3.0\% $(n=2)$ felt it lowered their grades while 15.2\% $(n=10)$ felt that methylphenidate did not have any influence. Approximately a third of methylphenidate users $(n=24 ; 36.4 \%)$ obtained methylphenidate from a source other than a medical practitioner (Table 4).

\section{Co-use of alcohol and methylphenidate}

Of the past-year methylphenidate users, 16 (24.2\%) had ever used methylphenidate prior to drinking alcohol. Fifteen of these students indicated frequency: two (13.3\%) practised co-use more than once a month and one participant (6.7\%) admitted to co-use two to four times per week. Of the 15 cases, 10 (66.7\%) had experienced negative effects, mainly becoming intoxicated more easily (seven of the 10 cases).

\section{Discussion}

The current study is limited by the retrospective nature of the data and the use of self-reported data rather than formal diagnostic evaluation. No attempt was therefore made to estimate the prevalence of ADHD in the study group and the study focused on the prevalence of methylphenidate use. The use of junior residence inhabitants means that the results are not representative of all students at the UFS. Sixty-six participants (11.3\%) reported past-year use of methylphenidate. The overall prevalence is very similar to the findings of Jain et al. ${ }^{15}$ regarding current methylphenidate use among medical students at the UFS. Our results, however, show a relatively lower rate among students in Health Sciences and higher rates for students in Natural and Agricultural Sciences, Humanities and Law. The overall rate of use is considerably lower than the $17.2 \%$ recently reported by Steyn ${ }^{16}$ for an unidentified South African university.

The strong male preponderance in methylphenidate use coincides with a higher prevalence of ADHD in males. ${ }^{1}$ The mode age for the onset of methylphenidate use of 18 years suggests that students are introduced to cognitive enhancers either in Grade 12 or in the first year at university. Hereafter, methylphenidate use declines with advancement in study years.

Almost all methylphenidate users in the current study used it to improve their concentration, and 9 out of 10 of these students believed that the drug improved their academic performance. The fact that most indicated that they only used the drug prior to assessments and not every day is suggestive of use for the purpose of cognitive enhancement, rather than for control of ADHD symptoms. Linssen et al. ${ }^{17}$ did an extensive review of studies on cognitive enhancement by methylphenidate in healthy individuals and reported enhanced but differential effects on distinct cognitive functions. Methylphenidate did enhance, in descending order, working memory; processing speed; verbal learning and memory; attention and vigilance; and reasoning and problem solving in psychological test situations. It had no influence on visual learning and memory, though. The authors warned that the cognitive enhancement effect might 
not be visible when tested in more complex authentic situations, and that the effect size is unlikely to exceed that of sensible lifestyle choices like adequate sleep.

Short-term advantages of methylphenidate may, however, come at the expense of long-term retrieval. Exposure of the developing normal brain to methylphenidate may in particular enhance concentration and focus in the short term, yet at the cost of neural plasticity involving working memory. ${ }^{18}$ These changes may manifest in functional deficits, such as adapting to interpersonal exchange and group dynamics; impaired cognitive flexibility in dynamic situations such as driving; and insufficient behavioural flexibility to, for instance, recover from drug abuse. A further concern is that, in a normal healthy brain, dopamine and noradrenalin levels can easily be pushed beyond the optimal levels, disrupting attention, locomotor and impulse control. ${ }^{18}$

The percentage of methylphenidate users who obtained methylphenidate without a legal prescription in this study (36.4\%) is very similar to the results reported by Steyn. ${ }^{16}$ The figures of $27.3 \%$ of past-year users of methylphenidate with a diagnosis of ADHD, and $66.7 \%$ obtaining their methylphenidate through a prescription from a general practitioner or specialist are similar to the figures reported by Jain et al. ${ }^{15}$ for medical students at the UFS (30.2\% and $70.6 \%$, respectively). Such discrepancy indicates that there is a considerable degree of offlabel prescribing of the drug to healthy persons, presumably for cognitive enhancement. It should be noted that the $27.3 \%$ refers only to students indicating past-year use of methylphenidate. The figure represents $3.1 \%$ of the total study population.

Off-label prescribing is not illegal in South Africa, yet prescription is not based on the registration conditions of the drug, but rather on the judgement of the prescriber. ${ }^{19}$ As such, the prescriber is personally taking responsibility for harm caused by the particular medication. A study ${ }^{20}$ done on the practices of GPs in the Free State concerning methylphenidate showed that almost half of a sample of GPs considered the parents of ADHD patients as being 'difficult'. It is therefore possible that coercion plays a role in prescribing methylphenidate.

The results of the current study also revealed that considerable diversion of prescriptions for methylphenidate occurs, with $31.8 \%$ of the participants indicating that they obtain their supply from friends (without payment). Diversion of prescriptions is illegal ${ }^{21}$ and a potentially dangerous action as the user is exposed to the drug without screening for contra-indications and without proper counselling with regard to potential side effects and interactions.

Illegal trading is also taking place with $6.1 \%$ of participants buying methylphenidate from illegal sources. This is substantially lower than the results reported by Steyn. ${ }^{16}$ In addition to the risks involved in diversion of prescriptions, stock bought from illegal sources bears the risk of questionable origin and quality control as well as adulterants. Both diversion and illegal outlets are criminal offences and may lead to prosecution. According to the Drugs and Drug Trafficking Act No. 140 of 1992,21 possession of a dependence-inducing drug such as methylphenidate bought from sources other than the legal supply chain in South Africa is a crime punishable by a fine or imprisonment for up to 10 years. It is a matter of concern that, despite the apparently tight control measures suggested by the high scheduling of the drug, the law is easily ignored, probably due to lack of enforcement.
Nearly a quarter of past-year methylphenidate users in the current study have used methylphenidate with alcohol. Methylphenidate and alcohol do interact, increasing the risk of stimulant toxicity, which manifests mainly as cardiac stimulation and neuropsychiatric emergencies.22 Reasons for mortality in such cases include cardiovascular incidents, convulsions and suicide. Deaths related to alcohol intake in the presence of high methylphenidate levels, as well as an increase in dependence potential, may be related to the increased production of ethylphenidate, a metabolite that is formed when methylphenidate is metabolised in the presence of alcohol. ${ }^{23}$ The heightened sensitivity caused by co-use of alcohol and methylphenidate is also thought to increase the risk for dependency, and is particularly relevant for persons with a history of drug and/or alcohol dependency. ${ }^{22}$

The authors recommend that prescribers of methylphenidate should be enabled to diagnose and manage ADHD in the interest of enhanced access to treatment. This can be done through curricular changes in undergraduate programmes, combined with continued medical education.

Students and the greater general public need to be informed about the legal aspects of prescriptions and encouraged to report transgressions. Orientation of new students should include awareness of cognitive enhancer abuse and the potential dangers thereof.

\section{Conclusion}

The prevalence of $11.3 \%$ for methylphenidate use among students residing in junior on-campus residences at the UFS occurs against the background of a low prevalence of formally diagnosed cases of ADHD. Methylphenidate users in this study indicated that the basic need driving their use of methylphenidate is the need to concentrate, focus and calm down. Instances of co-use of methylphenidate and alcohol indicate a lack of knowledge of the medication involved, rather than deliberate misuse.

Acknowledgements - The authors wish to thank all participants in the study and Mr FC van Rooyen, Department of Biostatistics, Faculty of Health Science, UFS, for statistical support.

Ms T Mulder, medical editor, School of Medicine, UFS, is thanked for technical and editorial preparation of the manuscript.

\section{ORCID}

L Fechter (D) http://orcid.org/0000-0001-6028-3653

J Griesel (iD http://orcid.org/0000-0001-9495-4603

\section{References}

1. Centers for Disease Control and Prevention (CDC). Attention-deficit/ hyperactivity disorder. [last updated 2016 Oct 19; cited 2016 Dec 7]. Available from: http://www.cdc.gov/ncbddd/adhd.

2. Lara C, Fayyad J, de Graaf R, et al. Childhood predictors of adult attention-deficit/hyperactivity disorder: results from the World Health Organization World Mental Health Survey Initiative. Biol Psychiatry. 2009;65(1):46-54. doi:10.1016/j.biopsych.2008.10.005.

3. Fayyad J, De Graaf R, Kessler R, et al. Cross-national prevalence and correlates of adult attention-deficit hyperactivity disorder. Brit J Psychiatry. 2007;190:402-9. https://doi.org/10.1192/bjp bp.106.034389

4. Perold M, Louw C, Kleynhans S. Primary school teachers' knowledge and misperceptions of attention deficit hyperactivity disorder (ADHD). S Afr J Educ. 2010;30(3):457-73. 
5. Bolea-Alamañac B, Nutt DJ, Adamou $M$, et al. Evidence-based guidelines for the pharmacological management of attention deficit hyperactivity disorder: update on recommendations from the British Association for Psychopharmacology. J Psychopharmacol. 2014;28(3):179-203. doi:10.1177/0269881113519509.

6. Schachter HM, Pham B, King J, et al. How efficacious and safe is short-acting methylphenidate for the treatment of attentiondeficit disorder in children and adolescents? A meta-analysis CMAJ. 2001;165(11):1475-88.

7. Faraone SV, Wilens TE. Effect of stimulant medications for attentiondeficit/hyperactivity disorder on later substance use and the potential for stimulant misuse, abuse, and diversion. J Clin Psychiatry. 2007;68(Suppl 11):15-22. doi:10.4088/jcp.1107e28.

8. Morton WA, Stockton GG. Methylphenidate abuse and psychiatric side effects. Prim Care Companion J Clin Psychiatry. 2000;2(5):159-64. https://doi.org/10.4088/PCC.v02n0502

9. Republic of South Africa. Medicines and related substances control act; 1965 (Act No. 101 of 1965).

10. Meyers RL. The 100 most important chemical compounds: A reference guide. Westport: Greenwoods Press; 2007.

11. Truter I, Kotze TJVW. Prescribing patterns of methylphenidate in a South African patient population who are members of a private medical aid. Health SA Gesondheid. 2005;10(4):75-84.

12. Teter CJ, McCabe SE, Boyd CJ, et al. Illicit methylphenidate use in an undergraduate student sample: prevalence and risk factors. Pharmacotherapy. 2003;23(5):609-17. doi:10.1592/ phco.23.5.609.34187.

13. University of the Free State. Policy and additional regulations concerning the placement of students in junior residences, Main Campus. 2011. [cited 2016 Dec 7].

14. University of the Free State. Policy and additional regulations concerning the placement of students in senior residences, Main Campus. 2011. [cited 2016 Dec 7].
15. Jain R, Chang C, Koto M, et al. Non-medical use of methylphenidate among medical students of the University of the Free State. S Afr J Psychiat, 2017;23:a1006. doi:10.4102/sajpsychiatry. v23.1006.

16. Steyn F. Methylphenidate use and poly-substance use among undergraduate students attending a South African university. S Afr J Psychiat. 2016;22(1):a760. doi:10.4102/sajpsychiatry.v22i1.760.

17. Linssen AM, Sambeth A, Vuurman EF, et al. Cognitive effects of methylphenidate in healthy volunteers: a review of single dose studies. Int J Neuropsychopharmacol. 2014;17(6):961-77. doi: 10.1017/S1461145713001594.

18. Urban KR, Gao WJ. Performance enhancement at the cost of potential brain plasticity: neural ramifications of nootropic drugs in the healthy developing brain. Front Syst Neurosci. 2014;13:38. doi:10.3389/ fnsys.2014.00038.

19. Jansen RM. The off-label use of medication in South Africa-What about some information for medical practitioners? S Afr Med J. 2009;99(6):438-39.

20. Venter A, van der Linde GP, Joubert G. Knowledge, attitudes and practices of general practitioners in the Free State regarding the management of children with attention deficit hyperactivity disorder (ADHD). SA Fam Pract. 2003;45(5):12-7.

21. Republic of South Africa. Drugs and drug trafficking act, 1992 (Act No. 140 of 1992).

22. Barrett SP, Pihl RO. Oral methylphenidate-alcohol co-abuse. J Clin Psychopharmacol. 2002;22(6):633-34. doi:10.1097/00004714200212000-00020.

23. Markowitz JS, DeVane CL, Boulton DW, et al. Ethylphenidate formation in human subjects after the administration of a single dose of methylphenidate and ethanol. Drug Metab Dispos. 2000;28(6):62024.

Received: 10-10-2016 Accepted: 05-02-2017 